Bundesgesundheitsbl 2017 · 60:1317-1318

https://doi.org/10.1007/s00103-017-2644-4

๑) Springer-Verlag GmbH Deutschland 2017

CrossMark

\section{Joseph Kuhn' · Anke-Christine Saß ${ }^{2}$}

' GE 4.2 - Gesundheitsberichterstattung, Sozialmedizin, Öffentlicher Gesundheitsdienst, Bayerisches Landesamt für Gesundheit und Lebensmittelsicherheit, Oberschleißheim, Deutschland

${ }^{2}$ Robert Koch-Institut, Berlin, Deutschland

\title{
Gesundheit: Die Region im Fokus
}

"In health care, geography is destiny." (John E. Wennberg)

Die Betrachtung räumlicher Unterschiede bei Gesundheitsmerkmalen steht gewissermaßen schon an der Wiege der Epidemiologie: Wer kennt nicht die Kartierung der Cholerafälle in London rund um die Broad Street durch John Snow 1854, mit der er eine Wasserpumpe als Ausgangsort der Erkrankungsfälle identifizierte. Noch 100 Jahre älter ist die Idee Johann Peter Franks, durch Ärzte medizinische Topographien erstellen zu lassen, um regionale Eigenheiten im Krankheitsgeschehen und die damit verbundenen Lebensumstände zu beschreiben. Franks Aufforderung war ausgesprochen wirkmächtig, medizinische Topographien wurden im 18. und 19. Jahrhundert in ganz Europa und darüber hinaus erstellt. Zumindest indirekt war auch hier der Nutzen eines Vergleichs zwischen den Regionen mitgedacht.

Dies ist eine der Traditionslinien der Dokumentation kleinräumiger Unterschiede der Gesundheit, wie sie seit Langem zum Beispiel in der Gesundheitsberichterstattung (GBE) der Bundesländer durchgeführt wird. Dafür wird ein gemeinsamer Indikatorensatz für die Gesundheitsberichterstattung der Länder genutzt. Wichtige Eckdaten, von der Sterblichkeit bis zum Gesundheitspersonal, werden im Indikatorensatz der Länder auf der Ebene der kreisfreien Städte und Landkreise dargestellt. So spiegelt sich beispielsweise der $\mathrm{Zu}$ sammenhang zwischen der sozialen Lage und der Sterblichkeit auch geographisch wider: Regionen, die ökonomisch weniger gut dastehen, z. B. aufgrund überdurchschnittlicher Arbeitslosigkeit, weisen oft eine höhere Sterblichkeit auf.
Ein Teil der regionalen Unterschiede, die in der Gesundheitsberichterstattung dokumentiert werden, haben auch unmittelbare Versorgungsrelevanz. So haben etwa südbayerische Landkreise niedrigere Masernimpfraten als nordbayerische Landkreise, ein Befund, der für die Schwerpunktsetzung bei Impfkampagnen genutzt wird. Seit einigen Jahren kommen in der Gesundheitsberichterstattung, aber z. B. auch bei den Krebsregistern und anderen Datenhaltern, Gesundheitsatlassysteme dazu, die Regionaldaten kartografisch abbilden und so auch geografische Muster gesundheitlicher Merkmale schneller erkennen lassen.

In den letzten Jahren ist das Potenzial kleinräumiger Vergleiche zunehmend auch in der Versorgungsforschung selbst erkannt worden, z. B. für Fragen nach der Validität von Diagnosen, regionalen Unterschieden der Versorgungsqualität oder der Gewährleistung der rechtlich vorgegebenen Sicherstellung gleichwertiger Lebensbedingungen in allen Regionen. Dabei ist eine Vielzahl interessanter Ergebnisse zutage getreten. Beispielsweise gibt es auf Kreisebene große regionale Unterschiede in der Häufigkeit von Aufmerksamkeitsdefizit-/ Hyperaktivitätsstörung(ADHS)-Diagnosen, bei Operationen zur Entfernung der Gaumenmandel bei Kindern und Jugendlichen, in der Häufigkeit von Antibiotikaverordnungen, Kaiserschnitten und Amputationen bei Diabetes. Auch wenn oft nicht bekannt ist, was dabei die richtige Rate oder eine Versorgungsnorm wäre, machen die regionalen Unterschiede bei solchen Versorgungsindikatoren deutlich, dass dahinter nicht nur regional unterschiedliche Erkrankungshäufigkeiten stehen können. Dafür sind die regionalen Varianzen häufig zu groß. Sie geben vielmehr Hinweise darauf, dass im Versorgungsalltag je nach Region unterschiedlich gehandelt wird und daher die Versorgungsleistungen, die Patienten bekommen, vielfach in erheblichem Ausmaß vom Wohnort abhängen. Dies ist gesundheitspolitisch handlungsrelevant, sowohl unter dem Gesichtspunkt der Versorgungsgerechtigkeit als auch der nach Art. 72 (2) des Grundgesetzes und den daran anschließenden raumordnungsrechtlichen Bestimmungen anzustrebenden gleichwertigen Lebensverhältnissen im Bundesgebiet.

Was die Verfügbarmachung kleinräumiger Datenanalysen für die Diskussion um die Versorgungsqualität angeht, haben u.a. das Zentralinstitut für die Kassenärztliche Versorgung in Deutschland mit dem Versorgungsatlas (www.versorgungsatlas.de) und die Bertelsmann Stiftung mit dem Faktencheck Gesundheit (https://faktencheckgesundheit.de) ebenso Pionierarbeit geleistet wie die kleinräumigen Analysen einiger Krankenkassen. Inzwischen gibt es zahlreiche Datenquellen, die für regionale Auswertungen erschlossen wurden. $\mathrm{Zu}$ verschiedenen Gesundheitsaspekten liegen empirische Regionalanalysen vor und es hat sich - etwa über den Arbeitskreis für medizinische Geographie in der Deutschen Gesellschaft für Geographie (www.med-geo.de) - eine eigenständige und über die klassische geografische Statistik hinausgehende Methodendiskussion zu diesem Themenfeld entwickelt. Ein Ergebnis ist beispielsweise die Gute Kartographische Praxis im Gesundheitswesen, eine Leitlinie zur kartografischen Darstellung von Regionaldaten. 


\section{Editorial}

Kleinräumige, auf die Versorgungsqualität bezogene Analysen stehen im Mittelpunkt dieses Schwerpunkthefts. Sie liefern nicht nur den unmittelbaren Leistungsträgern im Gesundheitssystem wichtige Erkenntnisse, sondern gewinnen durch die neuerdings wieder stärker diskutierte Notwendigkeit örtlicher Koordinierung zunehmend an Bedeutung. Anwendungsbeispiele sind unter anderem die kommunalen Gesundheitskonferenzen, die Altenhilfeplanung oder die Umsetzung des Präventionsgesetzes sowie die Bemühungen um eine regional differenzierte Bedarfsplanung auch für andere Akteure.

Das Schwerpunktheft stellt exemplarisch empirische Befunde aus der Forschung zu kleinräumigen regionalen Unterschieden der Gesundheit, aktuelle konzeptionelle Überlegungen zum Nutzen kleinräumiger Vergleiche für die Gesundheitsversorgung und zu methodischen Herangehensweisen vor. Ein eigenes Thema wären Vergleiche auf der Ebene der Bundesländer bzw. der internationalen Ebene. Beispielsweise wendet die Europäische Union die offene Methode der Koordinierung (OMK) nach Art. 5 AEU-Vertrag insbesondere auch in der Gesundheitsversorgung an. Hier sind ihre Rechtssetzungsbefugnisse sehr begrenzt. Die OMK ist darauf ausgerichtet, durch ein Monitoring regionaler Unterschiede der Gesundheit auf der nationalen Ebene auf eine Harmonisierung der Entwicklung in der EU hinzuwirken. Internationale Vergleiche werfen aber beispielsweise durch die dabei zu berücksichtigenden Unterschiede der Gesundheitssysteme und der für die Länder verfügbaren Datenquellen eine Vielzahl ganz eigener Probleme auf, die in einem darauf konzentrierten Schwerpunktheft aufzugreifen wären.
Das Schwerpunktheft soll exemplarisch den erreichten Entwicklungsstand kleinräumiger Datenanalysen dokumentieren und der Diskussion um die damit verbundenen Handlungsperspektiven Impulse geben.

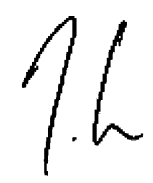

Joseph Kuhn

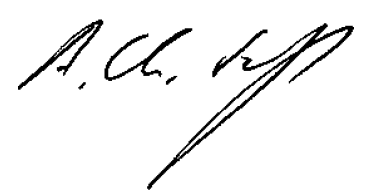

Anke-Christine Saß

\section{Korrespondenzadresse}

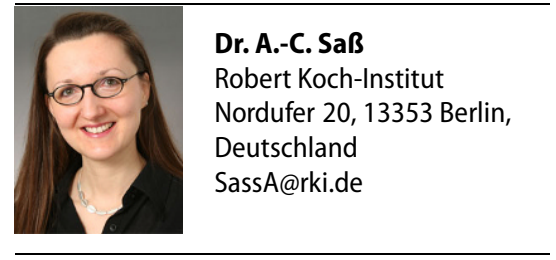

Interessenkonflikt. J. Kuhn und A.-C. Saß geben an, dass kein Interessenkonflikt besteht. 